Document downloaded from:

http://hdl.handle.net/10251/43405

This paper must be cited as:

Fajardo Peña, P.; Hoyas Calvo, S.; Gil Megías, A.; Pérez Quiles, MJ. (2014). Analysis of bifurcations in a Bénard-Marangoni problem: Gravitational effects. International Journal of Heat and Mass Transfer. 73:33-41. doi:10.1016/j.jhheatmasstransfer.2014.01.061.

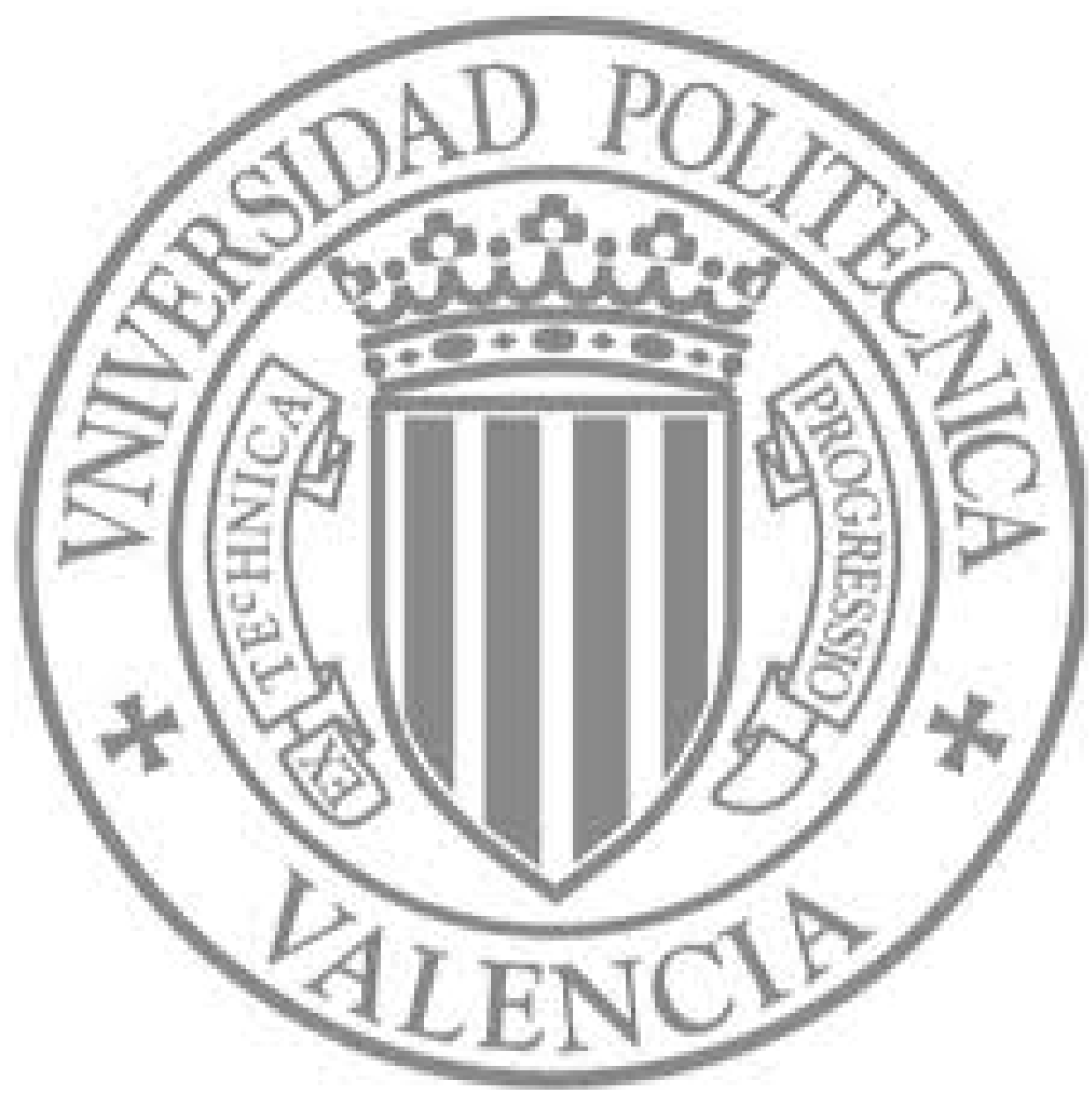

The final publication is available at

http://dx.doi.org/10.1016/j.ijheatmasstransfer.2014.01.061

Copyright Elsevier 


\title{
Analysis of Bifurcations in a Bénard-Marangoni Problem: Gravitational effects
}

\author{
S. Hoyas ${ }^{\mathrm{a}, *}$, P. Fajardo ${ }^{\mathrm{b}}$, A. Gila ${ }^{\mathrm{a}}$, M.J. Perez-Quiles ${ }^{\mathrm{c}}$ \\ ${ }^{a}$ CMT-Motores Térmicos, Universitat Politècnica de València, Valencia 46022, Spain. \\ ${ }^{b}$ Bioingeniería e Ingeniería Aeroespacial, Universidad Carlos III de Madrid, Leganés \\ 28911, Spain. \\ ${ }^{c}$ Instituto Universitario de Matemática Pura y Aplicada, Universitat Politècnica de \\ València, Valencia 46022, Spain.
}

\begin{abstract}
This article studies the linear stability of a thermoconvective problem in an annular domain for different Bond (capillarity or buoyancy effects) and Biot (heat transfer) numbers for two set of Prandtl numbers (viscosity effects). The flow is heated from below, with a linear decreasing horizontal temperature profile from the inner to the outer wall. The top surface of the domain is open to the atmosphere and the two lateral walls are adiabatic. Different kind of competing solutions appear on localized zones of the Bond-Biot plane. The boundaries of these zones are made up of co-dimension two points. A co-dimension four point has been found for the first time. The main result found in this work is that in the range of low Prandtl number studied and in low-gravity conditions, capillarity forces control the instabilities of the flow, independently of the Prandtl number.
\end{abstract}

Keywords: Thermocapillary convection, Linear stability, Gravitational Effects, Bond number

\section{Introduction}

It is well known that two different effects are responsible of the thermoconvective instabilities in fluid layers: gravity and capillarity forces. The problem in which both effects are considered, known as Bénard-Marangoni

${ }^{*}$ Corresponding author. e-mail: serhocal@mot.upv.es. Tel: +34 - 963877007 (ext $76563)$

Preprint submitted to International Journal of Heat and Mass Transfer February 7, 2014 
(BM) convection, has become a classical problem in fluid mechanics [1]. In the classical BM problem, heat is uniformly applied from the bottom wall and the solution becomes unstable for increasing temperature gradients. A more general problem includes the effect of non-zero horizontal temperature gradients arising new thermoconvective instabilities. These instabilities have been analyzed considering both a rectangular domain containing the flow $[29,19,11,26,6,3,20,24]$, or an annular geometries [14, 8, 15, 10, 21, 12].

In order to characterize the different effects steering the behavior of the flow, the following set of dimensionless numbers has been introduced:

1. Aspect ratio, $\Gamma=\delta / d$. Is the geometrical parameter that characterizes the domain.

2. Marangoni number, Mar $=\gamma \Delta T d^{2} / \rho \kappa \nu$ : Characterizes the surface tension effects.

3. Prandtl number, $\operatorname{Pr}=\nu / \kappa$ : The ratio of momentum diffusivity (kinematic viscosity) to thermal diffusivity. In this article several different Pr values will be considered covering the different situations concerning the momentum diffusivity to the thermal diffusivity.

4. Rayleigh number, $\mathrm{Ra}=g \alpha \Delta T d^{4} / \kappa \nu$ : Representative of the buoyancy effect.

5. Biot Number, Bi: Accounts for heat transmission between the fluid and the atmosphere. Values inside the range $[0.2-3.2]$ are explored in this article.

6. Bond number, Bo $=\mathrm{Ra} / \mathrm{Mar}=g \alpha \rho d^{2} / \gamma$ : Ratio of Rayleigh to Marangoni numbers, which is the control parameter in this analysis ranging from Bo $=0 \rightarrow g=0.0$ to $\mathrm{Bo} \approx 64 \rightarrow g=9.9$.

In the previous definitions, $\delta$ and $d$ are characteristic lengths of the domain that will be defined in the following section; $\gamma$ stands for the rate of change of surface tension with temperature; $\Delta T$ is the temperature increment, ranging from 2 to $50 ; \rho, \kappa, \alpha$ and $\nu$ are the density, the thermal diffusivity, thermal expansion coefficient and the kinematic viscosity of the fluid, respectively; and $g$ is the acceleration due to gravity.

The importance of heat-related parameters on the development of instabilities was analyzed in $[15,14]$. More recently, the problem was also studied in an annular geometry $[25,27,28]$ but neglecting the effect of Biot number and considering conduction through the lateral walls of the cylinder. Hoyas et al. [16] analyzed the effect of Biot number on the different bifurcations 
for the case of $\operatorname{Pr}=\infty$. The computational method was validated by comparing the results obtained with the experimental results by Garnier et al. [10]. The computational method has been recently modified [30] to be used with Prandtl numbers close to unity. In [12], the authors investigated the existence of co-dimension three bifurcations that are the points where the codimensions two curves intersect on the Prandtl-Biot plane and an also new kind of instability was predicted. Those latter works dealt with the influence of Biot number in the flow solutions. The interest in understanding the influence of gravitational effects in thermo-convective phenomena has been rapidly growing $[7,23]$. However, less attention has been paid to the effect of the capillarity forces of the onset of the flow motion and the behavior of the bifurcations that can appear.

The current work is devoted to obtain a deeper insight on the effect of the gravitational and capillarity forces of the onset of the flow motion, keeping in mind that understanding this flow behavior will contribute open a gateway to control the instabilities. To achieve this goal, a linear stability analysis, similar to the one in [16], will be performed, but instead of focusing on the influence of the Prandtl number, the focus will be put on understanding the effect of variations in the gravitational forces (or what is the same for a fixed geometry, the Bond number). Simulations will be performed in two different ranges of Prandtl number: $\operatorname{Pr}=1$ and $\operatorname{Pr}=50$. In [12] it was shown that $\operatorname{Pr}$

is the main parameter to determine the shape of the growing solution. This also applies to the current problem, but in the case of low gravity conditions, the dependency on Prandtl of the Rayleigh and Marangoni numbers is far less clear.

The paper is structured as follows. In the second section the formulation of the problem is presented, and in the third one the numerical method used to solve it. Then, in the fourth section the results are discussed. In the fifth and last section conclusions are presented, and future works are proposed. As this work contains many adimensional numbers and parameters, a list of symbols has been added before the bibliography.

\section{Model description and formulation}

The physical domain considered in this work consists of a horizontal fluid layer of depth $d$ ( $z$ coordinate) which is contained in the annular ring limited

by two concentric cylinders of radii a and $a+\delta$ ( $r$ coordinate). A sketch of the domain is presented in Fig. 1. The aspect ratio, $\Gamma$, is set to 4 and the 
diameters of the two cylinders are chosen so that the bigger is the double of the smaller one $(a=\delta)$. The bottom surface is considered to be rigid and is heated with a linear decreasing temperature gradient with a value of $T_{G}=2.2$ $\mathrm{K}$, which is kept constant throughout this study. The top surface is open to the atmosphere and the two lateral walls of the cylinder are considered adiabatic. The reference temperature used in the definition of the Rayleigh and Marangoni numbers is the mean temperature difference between the bottom plate and the atmosphere, $\Delta T$.

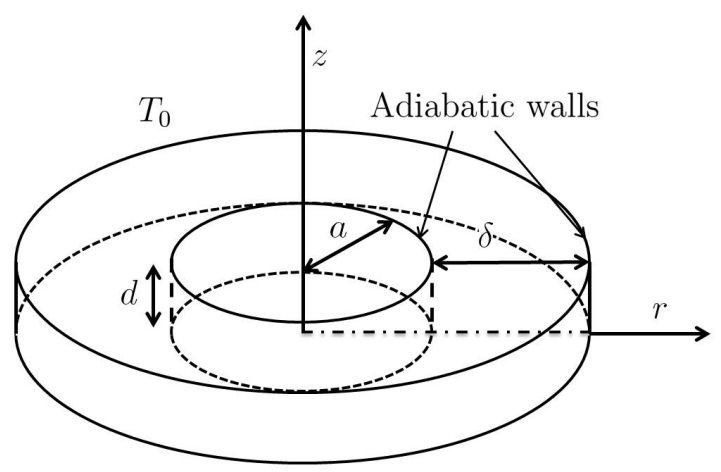

Figure 1: Sketch of the geometry. Lateral walls are considered adiabatic. The fluid is heated from below and the top surface is open to the atmosphere

The fluid layer behavior can be described by means of the momentum and mass balance equations and the energy conservation principle. These equations expressed in cylindrical coordinates and non-dimensionalized as in $[15,5]$ become

$$
\begin{aligned}
\nabla \cdot \mathbf{u} & =0 \\
\partial_{t} \mathbf{u}+(\mathbf{u} \cdot \nabla) \mathbf{u} & =\operatorname{Pr}\left(\nabla p+\nabla^{2} \mathbf{u}+\operatorname{Ra} \Theta \mathbf{e}_{z}\right) \\
\partial_{t} \Theta+\mathbf{u} \cdot \nabla \Theta & =\nabla^{2} \Theta .
\end{aligned}
$$

In the equations governing the system $u_{r}, u_{\theta}$ and $u_{z}$ are the components of the velocity field $\mathbf{u}, \Theta$ is the temperature, and $p$ is the pressure. In these equations the operators and fields are expressed in cylindrical coordinates and $\mathbf{e}_{z}$ is the unit vector in the $z$ direction. The Boussinesq approximation has been used as it is usual in this sort of problem [5]. Boundary conditions are similar to those of references $[15,30]$ and are summarized in Table 1. 
Briefly, the velocity is zero (no-slip wall condition) on the lateral walls and the bottom plate. On the top surface, the thermo-capillarity forces are modeled through the Marangoni condition [15], whereas for the heat transmission to the atmosphere is simulated by the Biot condition.

\begin{tabular}{|l|l|l|}
\hline$z=0$ & $z=d$ & $r=a, a+\delta$ \\
\hline \hline$u_{r}=0$ & $\partial_{z} u_{r}+\operatorname{Mar} \partial_{r} \Theta=0$ & $u_{r}=0$ \\
$u_{\phi}=0$ & $r \partial_{z} u_{\phi}+\operatorname{Mar} \partial_{\phi} \Theta=0$ & $u_{\phi}=0$ \\
$u_{z}=0$ & $u_{z}=0$ & $u_{z}=0$ \\
$\Theta=\Delta T-\left(T_{G} / \delta\right) r$ & $\partial_{z} \Theta+\operatorname{Bi} \Theta=0$ & $\partial_{n} \Theta=0$ \\
\hline
\end{tabular}

Table 1: Boundary conditions

As the temperature gradient is imposed, the fluid starts to evolve until a stationary state, commonly know as basic state, is reached. Due to the symmetries in the problem and given that the flow is laminar, the basic state can be approximated by a 2D axisymmetric solution. Therefore, the dependency with $\phi$ can be neglected. The equations then become:

$$
\begin{aligned}
r^{-1} \partial_{r}\left(r u_{r}\right)+\partial_{z} u_{z} & =0 \\
\operatorname{Pr}^{-1}\left(u_{r} \partial_{r} u_{r}+u_{z} \partial_{z} u_{r}\right) & =-\partial_{r} p+\Delta_{c} u_{r}-\frac{u_{r}}{r^{2}} \\
\operatorname{Pr}^{-1}\left(u_{r} \partial_{r} u_{z}+u_{z} \partial_{z} u_{z}\right) & =-\partial_{z} p+\Delta_{c} u_{z}+\operatorname{Ra} \Theta, \\
u_{r} \partial_{r} \Theta+u_{z} \partial_{z} \Theta & =\Delta_{c} \Theta,
\end{aligned}
$$

where $\Delta_{c}=r^{-1} \partial_{r}\left(r \partial_{r}\right)+\partial_{z}^{2}$ is the Laplacian operator expressed in cylindrical coordinates. To obtain the proper solution, the previous equations are supplemented with the boundary conditions gathered in Table 1.

\section{Numerical Method}

The previous system of equations was solved numerically using a collocation method [22]. The method starts by expanding the fluid variables in a truncated series of orthonormal Chebyshev polynomials, as

$$
p(r, z) \simeq \sum_{n=0}^{N} \sum_{m=0}^{M} a_{n m} T_{n}(r) T_{m}(z)
$$




$$
\begin{aligned}
& u_{r}(r, z) \simeq \sum_{n=0}^{N} \sum_{m=0}^{M} b_{n m} T_{n}(r) T_{m}(z), \\
& u_{z}(r, z) \simeq \sum_{n=0}^{N} \sum_{m=0}^{M} c_{n m} T_{n}(r) T_{m}(z), \\
& \Theta(r, z) \simeq \sum_{n=0}^{N} \sum_{m=0}^{M} d_{n m} T_{n}(r) T_{m}(z),
\end{aligned}
$$

where $T_{i}(x)$ is the Chebyshev polynomial of the first kind of degree $i$. The polynomial coefficients, $a_{n m}, b_{n m}, c_{n m}$, and $d_{n m}$, are now the unknowns of the problem. The flow variables expanded expressions are substituted into Eqs. (1), (2) and (3) and boundary conditions (Table 1). The resultant equations are then evaluated in the Chebyshev-Gauss-Lobatto (CGL) points [4],

$$
\begin{aligned}
& r_{i}=\cos \left(\pi \frac{i}{N}\right), j=0,1, \ldots, N, \\
& z_{i}=\cos \left(\pi \frac{i}{M}\right), j=0,1, \ldots, M,
\end{aligned}
$$

where $N$ and $M$ correspond to the order of the method in radial and axial direction, respectively. The use of CGL points is of interest when dealing with boundary effects [17] since the points are not equispaced but tend to concentrate near the boundaries. The boundary condition for the pressure is obtained projecting the equations by the normal to the boundaries and evaluating the projected equations there. The use of this procedure, as proposed in [18], avoided the problem of the spurious modes [2]. As the pressure is determined up to an additive constant, an arbitrary value for it is fixed in a boundary point.

The non-linearity of the problem was solved by using a Newton-like iterative method, taking as first contribution either a solution of the linearized problem (neglecting the nonlinearity of eq. (5) and eq. (6)) or a known basic state "close" to the new one. Typically convergence was obtained in less than 20 iterations as shown in the convergence test performed in [15].

As the Rayleigh number is increased (and thus Marangoni), the basic state becomes unstable and several different bifurcations arise. The purpose of the analysis is to determine the critical Rayleigh and Marangoni numbers values and thus the shape of growing instabilities for fixed Biot, Prandtl 
and Bond numbers. As previously stated, the analysis will be done for two extreme ranges of the Prandtl number to cover the different flow situations in terms of the relative importance of the momentum diffusivity to the thermal diffusivity.

The stability is analyzed by perturbing the basic solutions with fields depending on the three coordinates, $r, \phi$ and $z$. Using again that the problem is axisymmetric, and thus there is periodicity in $\phi$, fluid magnitudes may be expanded in Fourier modes in $\phi$ as

$$
X(r, \phi, z, t)=X_{b}(r, z)+X_{p}(r, z) e^{i k \phi+\lambda t}
$$

where subscript $b$ denotes the basic state and $k \geq 0$ is the wave number. The real part of the eigenvalue, $\lambda$, characterizes the instability. If it is negative the basic state is stable whereas if it is positive the basic solution is unstable. The imaginary part accounts for the stationarity of the given state. In this case, it can be either zero when the bifurcation is stationary, or non zero when it has an oscillatory behavior.

The eigenvalues and eigenfunctions of this problem are computed substituting the Fourier expansion (12) into the general equations (1-3) and the BCs from Table 1. After linearizing the problem by neglecting the non-linear terms, a generalized eigenvalue problem of the form

$$
A \bar{X}=B \lambda \bar{X}
$$

is obtained, as shown by [30].

The matrix B is singular, due to the presence of the boundary conditions, and thus not all the eigenvalues have a finite value. In the current work, a computational technique, developed by Navarro et al. [21], specifically designed for thermo-convective problems is used. The largest eigenvalue obtained through this transformation corresponds to the largest finite eigenvalue of the original problem. As shown in previous works [30, 12], several bifurcations may appear depending on the symmetries of the growing perturbation. Up to four different competing solutions for the different wave numbers have been found, namely: stationary roll (SR), similar to the ones of the basic state [29]; hydrothermal wave or oblique traveling waves (HWI) [29]; longitudinal rolls (LR) [3, 16]; and a standing hydrothermal wave of second class or flower-like wave (HWII) $[9,10]$. These solutions will be described later. 
All this formulation has been implemented in Fortran90, based in an earlier implementation [15] for infinity Prandtl numbers. The previous code was validated experimentally in [16]. The generalization to $\operatorname{Pr}<50$ was validated in [30], using the same procedure that in [15].

\section{Discussion}

\subsection{Basic state}

Neither the variation of the Biot nor the Prandtl numbers affects greatly to the shape of the basic state, being the Bond number the most significative parameter. In Fig. 2, the temperature profiles and velocity diagrams for extreme values of Bond numbers, $g=9.9$ (left) and $g=0.0$ (right), are plotted at their critical Rayleigh and Marangoni numbers. In both cases the Prandtl number is moderately low $(\mathrm{Pr}=0.7)$. In the case plotted at the left of Fig. 2, the Bond number is Bo $=68.44$ with $\mathrm{Ra}_{\mathrm{c}}=2113.7$ and $\operatorname{Mar}_{\mathrm{c}}=30.88$. The left plot is similar to those previously reported for $\operatorname{Pr}=\infty$ both experimentally and numerically $[14,16]$ and qualitatively similar to those used in Riley and Neitzel [26]. They are similar for all the range of Prandtl number studied (not shown). The velocity diagram presents two long co-rotative rolls. These rolls distribute the heat in convective way, supported by the couple between the energy and momentum equation trough the Rayleigh term. Things change completely in the second case plotted. There, Bo $=0.0$ with $\mathrm{Ra}_{\mathrm{c}}=0.0$ and $\mathrm{Mar}_{\mathrm{c}}=235.61$. Obviously, without gravity buoyancy effects are not important any more. The only thermal effects come from the Marangoni effect a the top of the container. This produce that the vertical component of the rolls be relatively smaller than in the buoyant-dominant case, creating an almost parallel stratification.

\subsection{Momentum diffusivity dominating flow $(\operatorname{Pr}=50)$}

In [12], the authors studied this problem but in buoyancy dominant conditions. They found there that the shape and class of the growing perturbation depends only on the Biot and Prandtl numbers. In this case the Bond number plays a critical role, changing the main cause of instabilities in the flow from gravitational forces to capillarity ones. The representative top $r-\phi$ plane isotherms corresponding to the different regions of the Biot-Bond plane are shown in Fig. 4. Two different regions, bounded by codimension two points appear. These points are defined by the condition that for the same critical pair $\left(\mathrm{Ra}_{\mathrm{c}}, \mathrm{Mar}_{\mathrm{c}}\right)$ two different competing solutions can be found. The shaded 

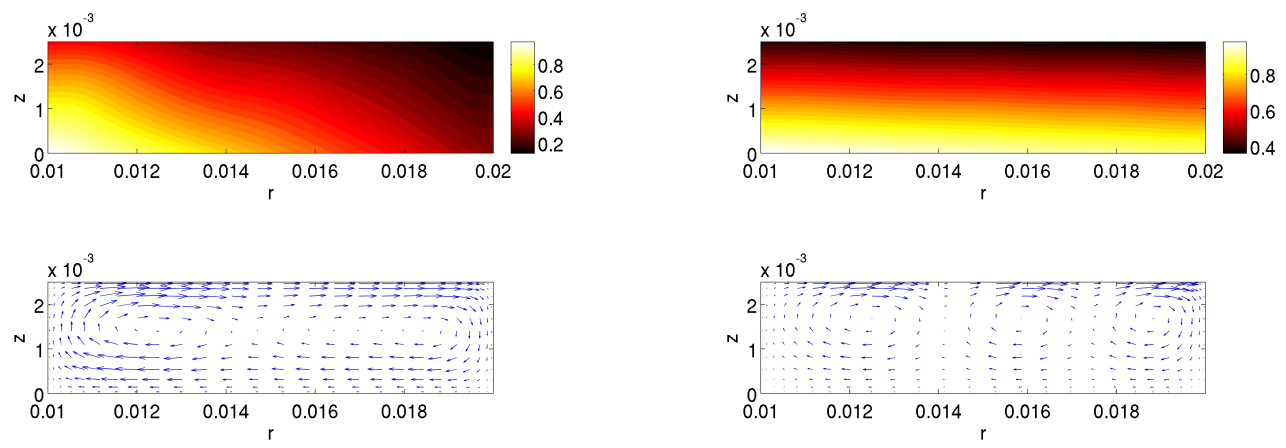

Figure 2: Temperature profile and velocity diagram of the basic state at their relative critical Rayleigh number for the highest (left) and lowest (right) bond numbers studied. The left figure corresponds to $\mathrm{Bo}=68.44, \mathrm{~g}=9.9$, and the right one to $\mathrm{Bo}=0.0, \mathrm{~g}=0.0$,

curve separates two regions: HWI, left, wave numbers ranging from 10 to 15, and longitudinal rolls, region LR, right, wave numbers from 13 to 19. The open symbols at the bottom right separate two different zones of the LR region. The $3 \mathrm{D}$ structure of these growing solutions is presented in Fig. 3 for a codimension two point around $\mathrm{Bi}=1.8$ and $\mathrm{Bo} \simeq 45$. In this case both solutions present a $r-z$ structure made up of two counter-rotative vortex. The boundary between these vortex is exactly at the same $r$ where the maximum heat is reached. Depending on the imaginary part of the eigenvalue with the maximum real part, this structure rotates ( Fig. 3a) or stay still (Fig. 3a). In the case of low Bond number, the lateral structure presents more co-rotative rolls, corresponding to the enlargement of the structures.

Below that curve, for low Bond numbers, wave numbers are around 16 and the rolls of the instabilities are close to the outer wall. Above the line, the wave number is around 13, being the rolls closer to the hotter inner wall. As we travel in the figure to regions of lower Biot and higher Bond, the wave number tends to 13 in both cases. This transition can be very hard to compute, as is shown in marginal stability diagram of Fig. 5. From $k=0$ to $k=12$ the highest eigenvalue is complex, and thus the growing perturbation is a hydrothermal wave (HWI). At $k=13$, the highest eigenvalue correspond to a longitudinal roll equivalent to the one shown in Fig. 4 above the open line. This curve has a very sharp shape since, for $k=12$, the eigenvalue 


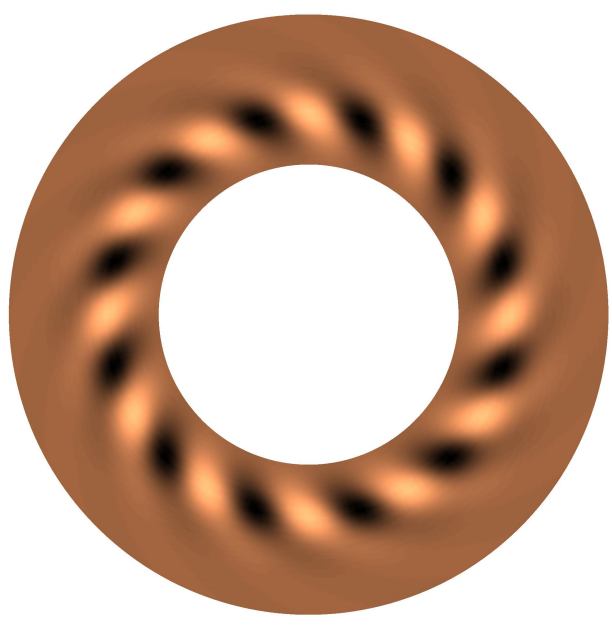

(a)

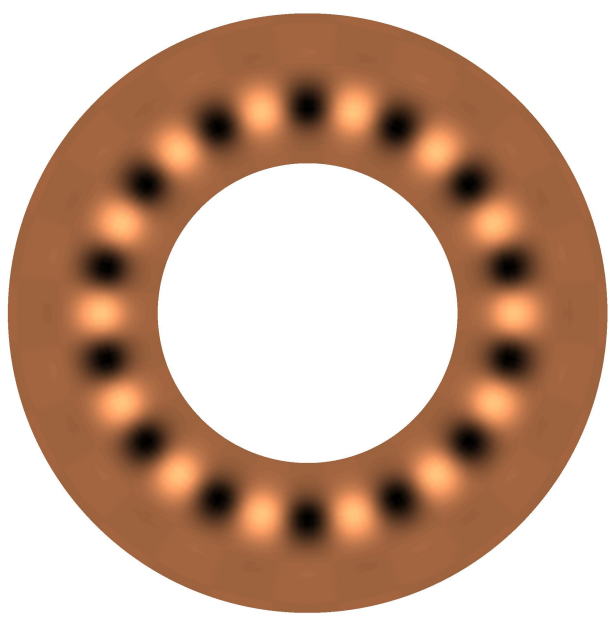

(c)
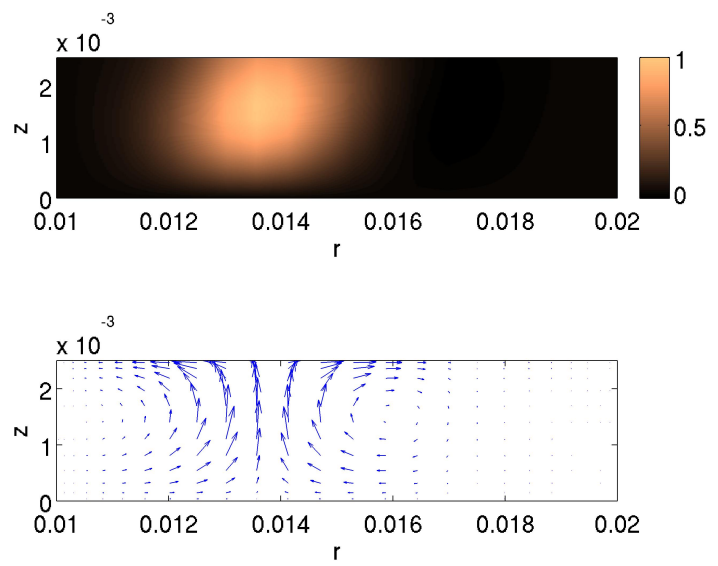

(b)
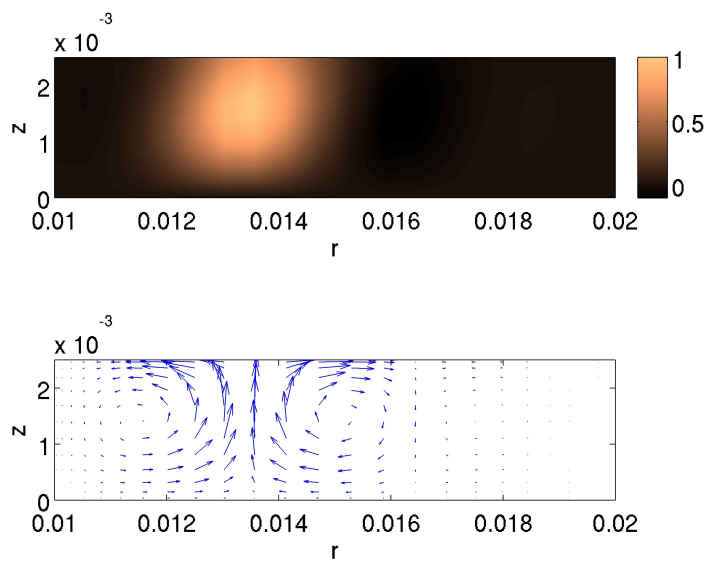

(d)

Figure 3: Temperature distribution (top and lateral view) and lateral velocity diagram of an hydrothermal wave of the first (a,b) class and a longitudinal roll $(\mathrm{c}, \mathrm{d})$ for $\operatorname{Pr}=50, \mathrm{Bi}=1.8$ and $\mathrm{Bo} \simeq 45$ in a codimension two point. Temperatures and velocities has been adimensionalized dividing them by its maximum 
corresponding to this solution is below -6 . The last curve in this figure corresponds to a solution in the LR region, below the empty dot line of Fig. 4. For $k>30$ the real part of the eigenvalues is below -2 .

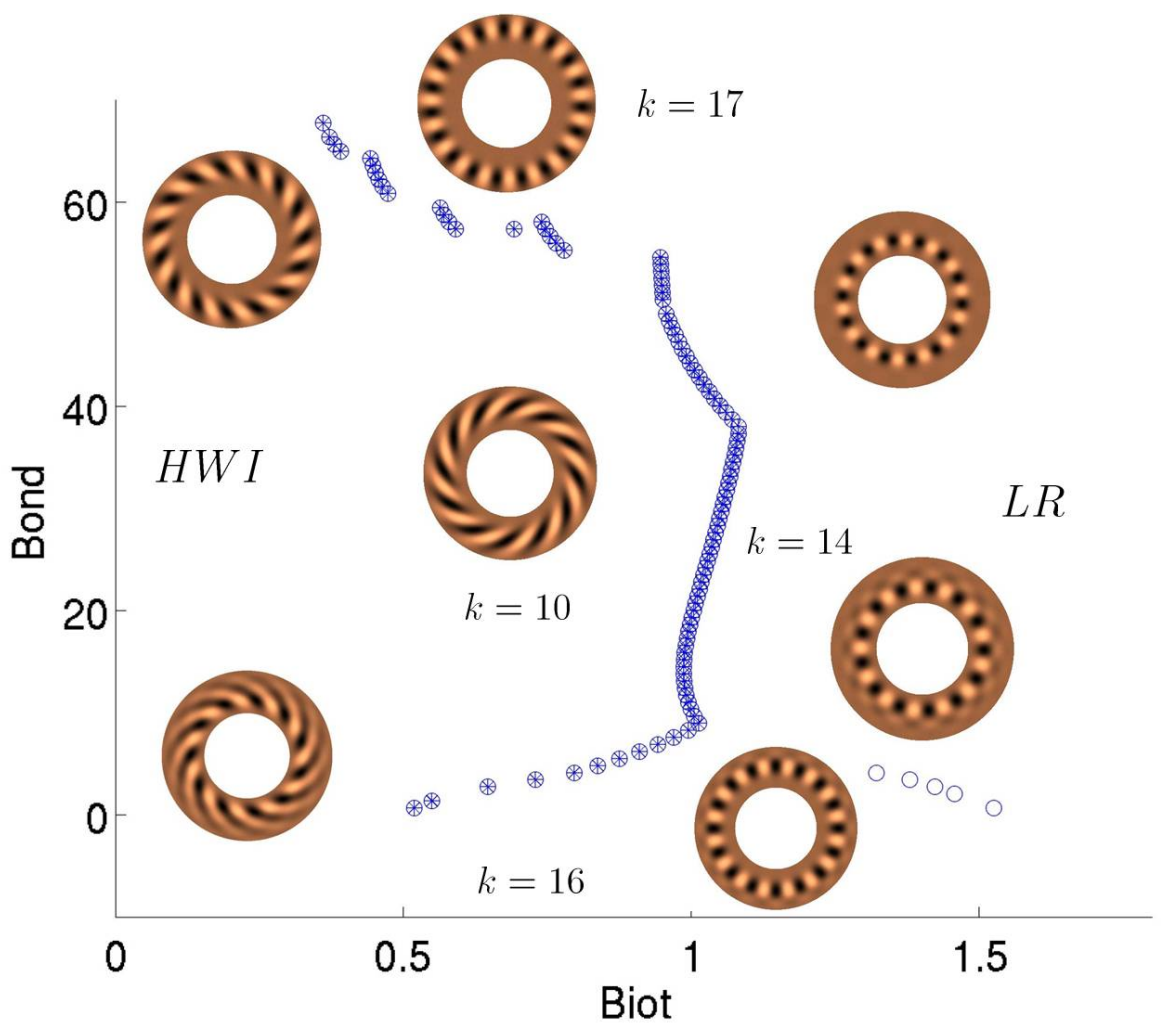

Figure 4: Bond-Biot plane stability diagram showing regions HWI and LR. Representative top $r-\phi$ plane isotherms corresponding to aforementioned regions are shown. The shaded points made up the boundary whereas the open ones separate two different zones of the LR region

One difficult question to answer is at what Bo the main responsible of the onset of the instabilities changes from capillarity forces to gravitational ones. In Fig. 6 the critical pairs $\left(\mathrm{Ra}_{c}, \mathrm{Mar}_{c}\right)$ have been plotted for different Biot and Bond numbers. It is well known $[15,12]$ that as Biot decreases the system becomes more stable and thus the values of $\mathrm{Ra}_{c}$ and $\mathrm{Mar}_{c}$ to destabilize it are higher. Focusing at the top curve of Fig. $6(\mathrm{a})$, for $\mathrm{B}=0.2$, as the Bond number is increased the curve follows a linear profile until approximately Bo $=13.8(g=2)$. The rate of decrease is similar for all the Biot numbers. 
Again, for $\mathrm{B}=0.2$ the critical $\left(\mathrm{Ra}_{c}, \mathrm{Mar}_{c}\right)$ for each Bond number, together with the critical $\Delta T$ has been plotted in Fig. 6(b). For Bond numbers over approximately 25, the critical Rayleigh numbers follow a straight pattern, indicating that the main cause of the instabilities for Bo $\geq 25$ is buoyancy. As it is also shown in this figure, the critical temperature $\Delta T_{c}$ decreases with increasing Bond numbers, showing that buoyancy is a more effective mechanism for destabilization of the flow. In the case of thermocapillarity, from Fig. 6 it seems to be the main effect for Bo $\leq 10$, whereas the transition from Marangoni to Rayleigh is produced between 10 and 25.

The idea stated in the previous paragraph can be reinforced with the information provided in Fig. 4. As we already said, as the Bond number is reduced, the system is able to store more heat and the critical temperature grows. This produces that the structures present in the flows get larger. As example of this can be see in the structures for case of $B i=0.2, B o=0$, at the bottom-left corner of Fig. 4. For small Biots, the structures are usually attached to the colder cylinder [16]. In this case, as we have neglected the buoyancy effects, the only responsible of this large structure has to be the Marangoni condition. 


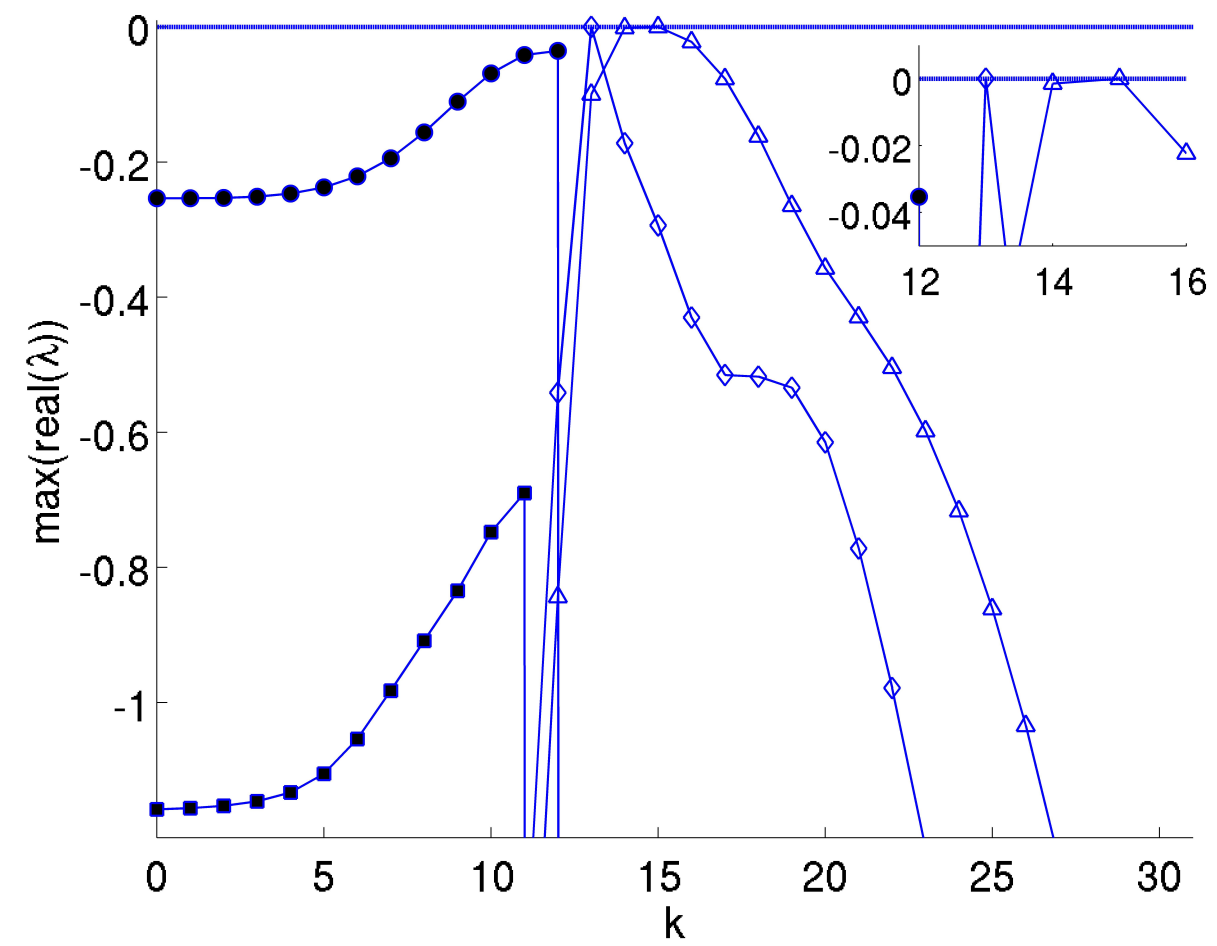

Figure 5: Marginal stability diagram of a co-dimension two bifurcation. $\mathrm{Ra}_{\mathrm{c}}=616.98, \mathrm{Mar}=148.72, \mathrm{Bi}=1.321, \mathrm{Pr}=50$. Empty points mean a real eigenvalue, whereas bold ones stand for complex eigenvalues. The curves group the eigenvalues corresponding to geometrically similar eigenfunctions. The value $\max \left|\operatorname{real}\left(\lambda_{13}, \lambda_{15}\right)\right| \leq 10^{-6}$. All these curves intersects transversally 


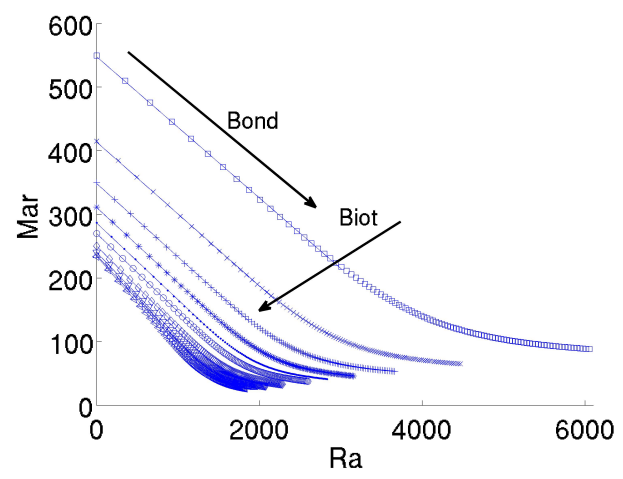

(a)

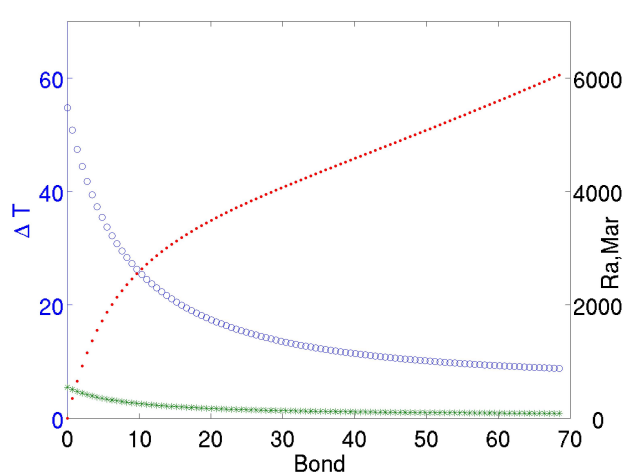

(b)

Figure 6: (a) Critical pairs $\left(\mathrm{Ra}_{c}, \mathrm{Mar}_{c}\right)$ for $\operatorname{Pr}=50$. Arrows mark the direction of increasing gravity, from 0.0 to 9.9 , with a 0.1 step, and of growing $\mathrm{B}$ for $0.2,0.3,0.4,0.5,0.6,0.7,0.9,1.1,1.3,1.5$. The lines approximate the points from $g=0.0$ to $g=2.0$ on least-squares sense. (b) Bond number dependency for $\mathrm{B}=0.2$ of Mar, Ra and $\Delta T$. Right axis: Critical Ra (dots) and Mar (stars). Left axis: $\Delta T$ (circles) 


\section{Prandtl numbers close to unity}

When $\operatorname{Pr}$ is close to unity, the nonlinearities of Navier-Stokes equations are of the same order of magnitude than the viscous terms. This increases the richness and complexity of bifurcations, making still harder to decide the point where the main effects are thermoconvective or thermocapillary. The lateral structure of a typical HWI solution is presented in 7, with several corotative rolls. This lateral structure is similar to the one of $8(\mathrm{~d})$. They are, however, completely different to the HWII, where a big structure expanding half of the domain is present. Moreover, in oder to fulfill the continuity equation, the $3 \mathrm{D}$ structure is most necessary. It is worth to say that this structure depends on buoyancy to exists.

The Bond-Biot plane can again describe properly the situation (Fig. 9). In this figure, codimension-two points for $\operatorname{Pr}=0.7$ circles, and $\operatorname{Pr}=1.2$, squares are shown. Representative top $r-\phi$ plane isotherms of the growing solution in several points of these plane has been plotted in Fig. 10. There are mainly five regions: Left top, stationary rolls (Fig.10a); Right top, hydrothermal waves of the second class (10d); Bond 40-60, Biot 0.5-1.5, Hydrothermal waves of the first class, $k=9$ (10c); Right bottom, Longitudinal rolls (Figs. 10f,10g,10k). When Pr is reduced, all the curves move rightward, to higher Biot numbers. This has also been seen for other Prandtl numbers computed (not shown). A very interesting solution is found for $\operatorname{Pr}=0.7$ at where a codimension four bifurcation takes place. This is the first time that a point like this has been found in this geometry.

The boundary between regions LR and HWI at the bottom-left of Fig. 9 presents a very curious characteristic. In Fig. 11 the location in the Ra-Mar plane of the codimension two-points has been plotted. The fan-like structure at the center of this figure corresponds to the boundary between region SR and HWI in Fig. 9. They resent a clear dependency on Pr. This is not true, or at least not so clear for the boundary between regions LR and HWI. These points follows a straight pattern that seems to be independent of Prandtl number. A possible explanation of this fact is that due to the relatively small Rayleigh number of this region, the nonlinear effects produced by the Prandtl number are not important.

From Fig. 9 it is also clear that changing properly Biot and Bond number it is possible to find any sort of bifurcation. Moreover, for Biot numbers below 1.5 it was found $[12,30]$ that the growing perturbation was always a stationary roll. Now it is clear that for at least $\operatorname{Pr}=0.7$ it is possible to 


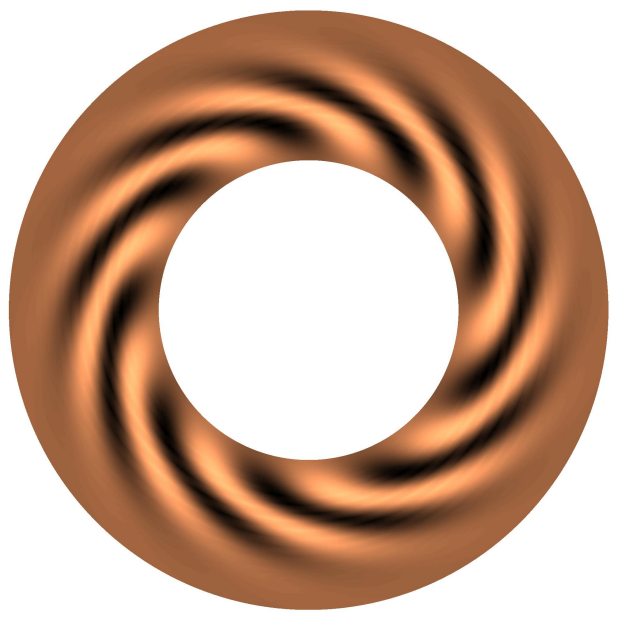

(a)
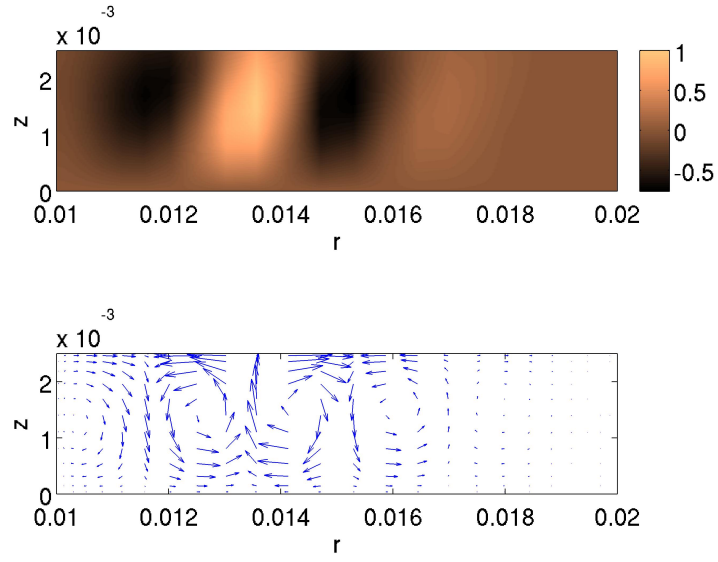

(b)

Figure 7: Temperature distribution and velocity diagram of an hydrothermal wave of the first class corresponding to point (c) of Fig. $9(\operatorname{Pr}=1.2, \mathrm{Bi}=1.2$ and $\mathrm{Bo} \simeq 60)$. Temperatures and velocities has been adimensionalized dividing them by its maximum 


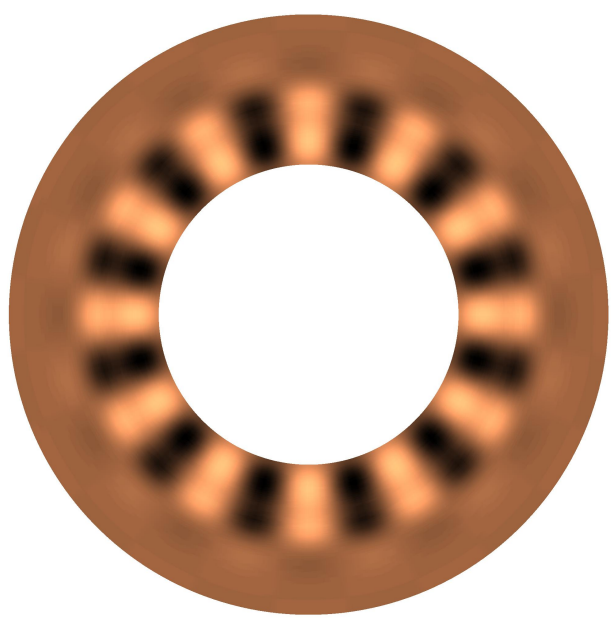

(a)

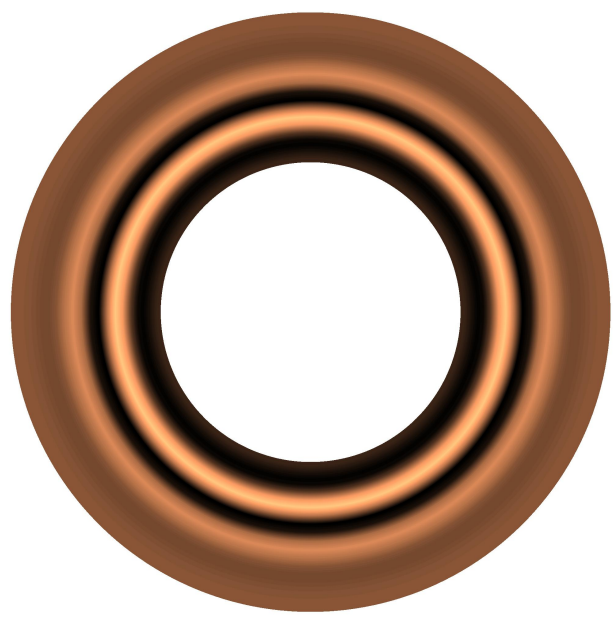

(c)
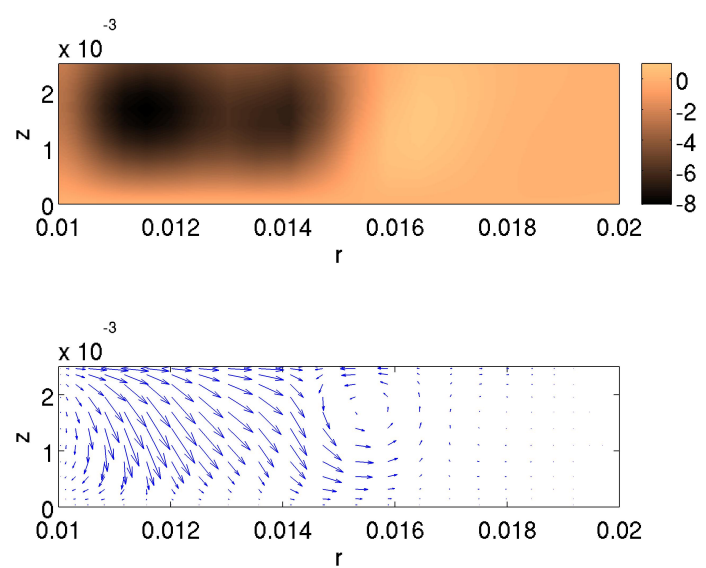

(b)
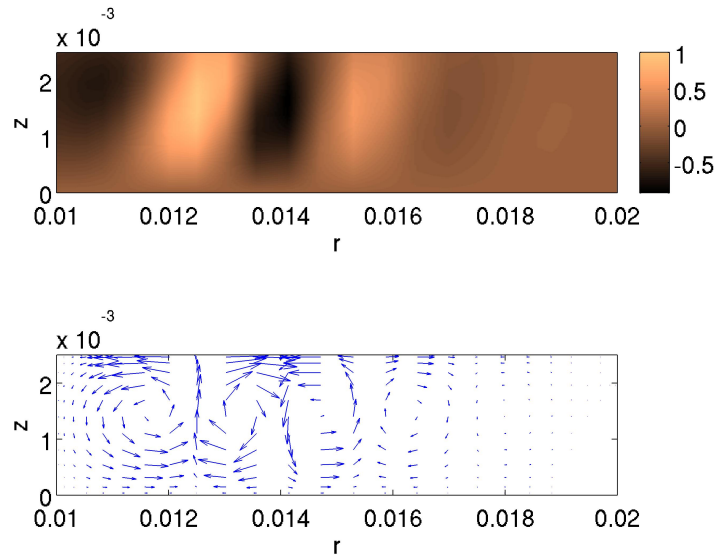

(d)

Figure 8: Temperature distribution and velocity diagram of an hydrothermal wave of the second class (a,b) and a stationary roll (c,d). The HWII corresponds to point (d) Fig. $9(\mathrm{Pr}=1.2, \mathrm{Bi}=1.8$ and $\mathrm{Bo} \simeq 60)$. The $\mathrm{SR}$ to point (a) $(\mathrm{Pr}=1.2, \mathrm{Bi}=0.2$ and $\mathrm{Bo} \simeq 30)$. Temperatures and velocities has been adimensionalized dividing them by its maximum 
obtain an hydrothermal wave, with a smaller value of the critical Rayleigh number.

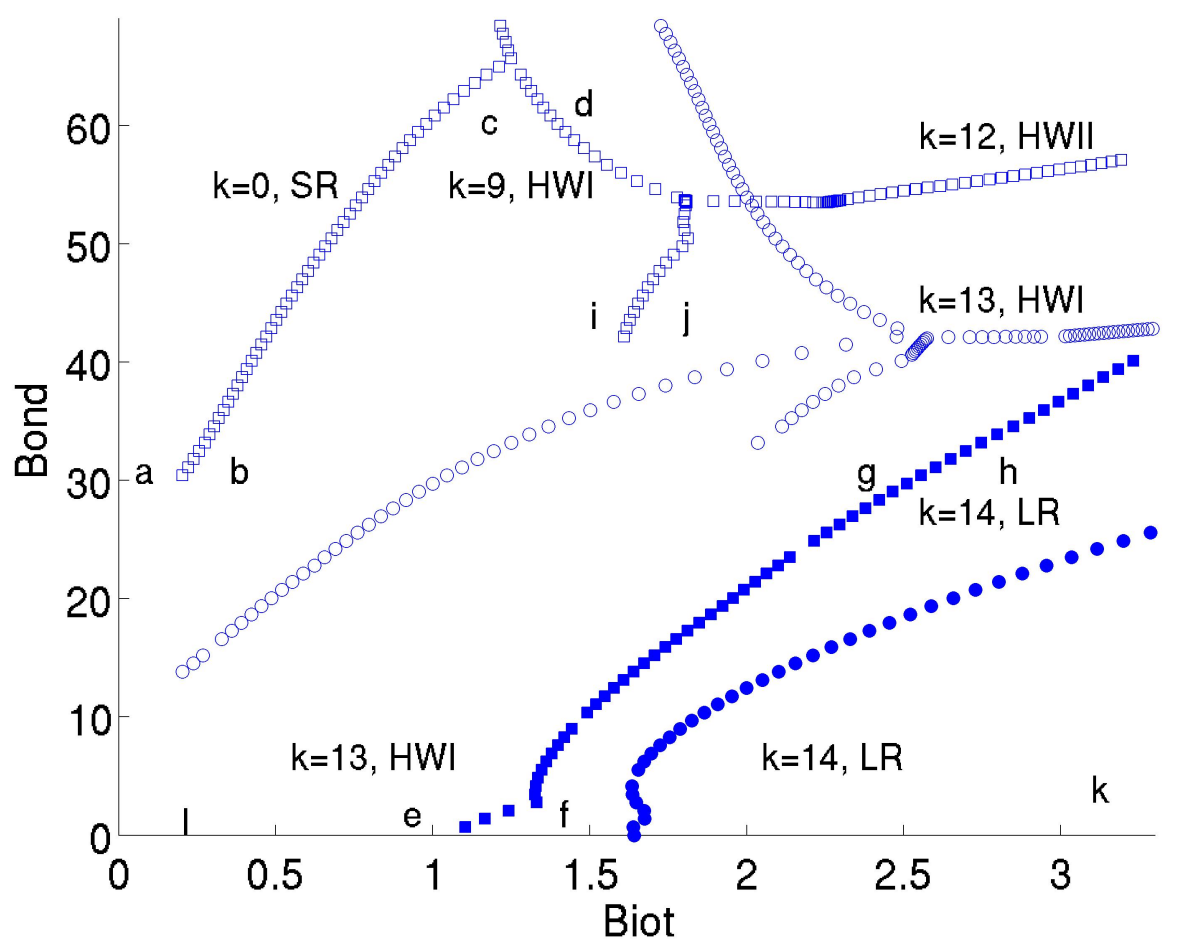

Figure 9: Bond-Biot plane stability diagram for $\operatorname{Pr}=0.7$, circles, and $\operatorname{Pr}=1.2$, squares. Bold points correspond to those in 11. The different regions found (HWI, HWII, SR and LR) for $\mathrm{Pr}=1.2$ are shown in the figure. Representative top $r-\phi$ isotherms for $\operatorname{Pr}=1.2$ in the approximated location of the points marked by letters are shown in Fig. 10

\section{Conclusions and future works}

In this work the instabilities appearing in a cylindrical annulus heated from below were analyzed by means of the linear stability method. This thermo- and fluid-dynamic problem is governed by mass, momentum and energy conservation equations in primitive variables, where the Boussinesq approximation has been used. Basic state and linear stability equations were 


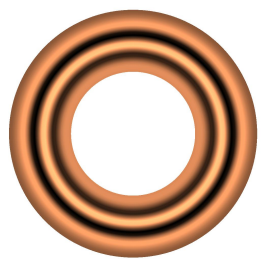

(a) SR, $B=0.22$

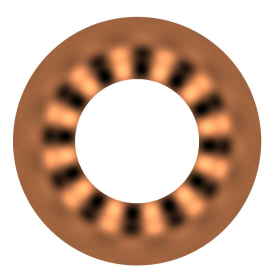

(d) HWII, $B=1.17$

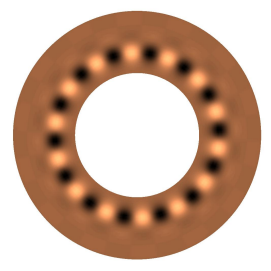

(g) LR, $B=2.6$

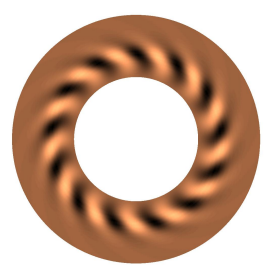

(j) HWI, $B=2.6$

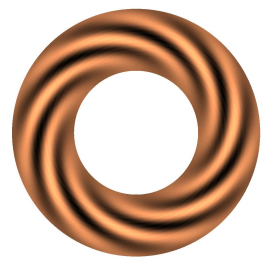

(b) HWI, $B=0.22$

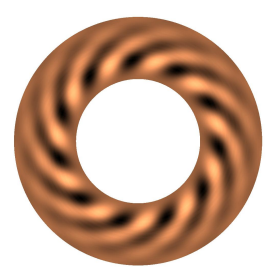

(e) HWI, $B=1.10$

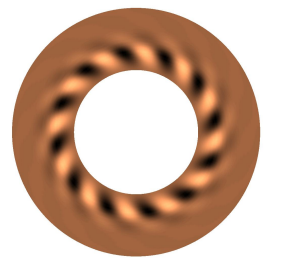

(h) HWI, $B=2.6$

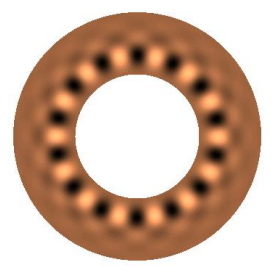

(k) LR, $B=3.1$

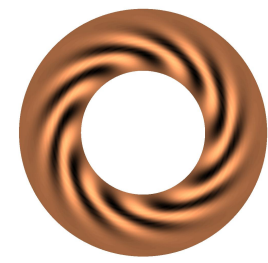

(c) HWI, $B=1.28$

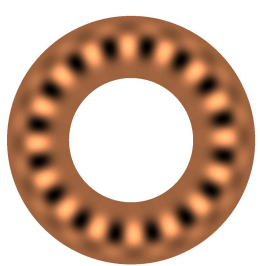

(f) LR, $B=1.10$

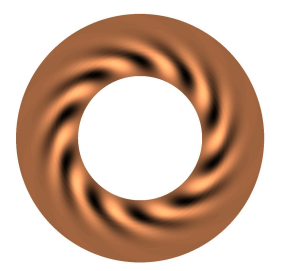

(i) HWI, $B=1.64$

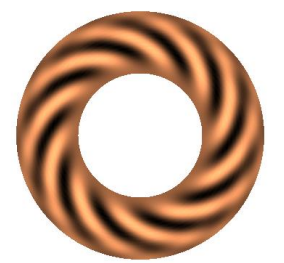

(l) HWI, $B=0.2$

Figure 10: Representative top $r \phi$ plane isotherms corresponding to the points marked in Fig. 9 


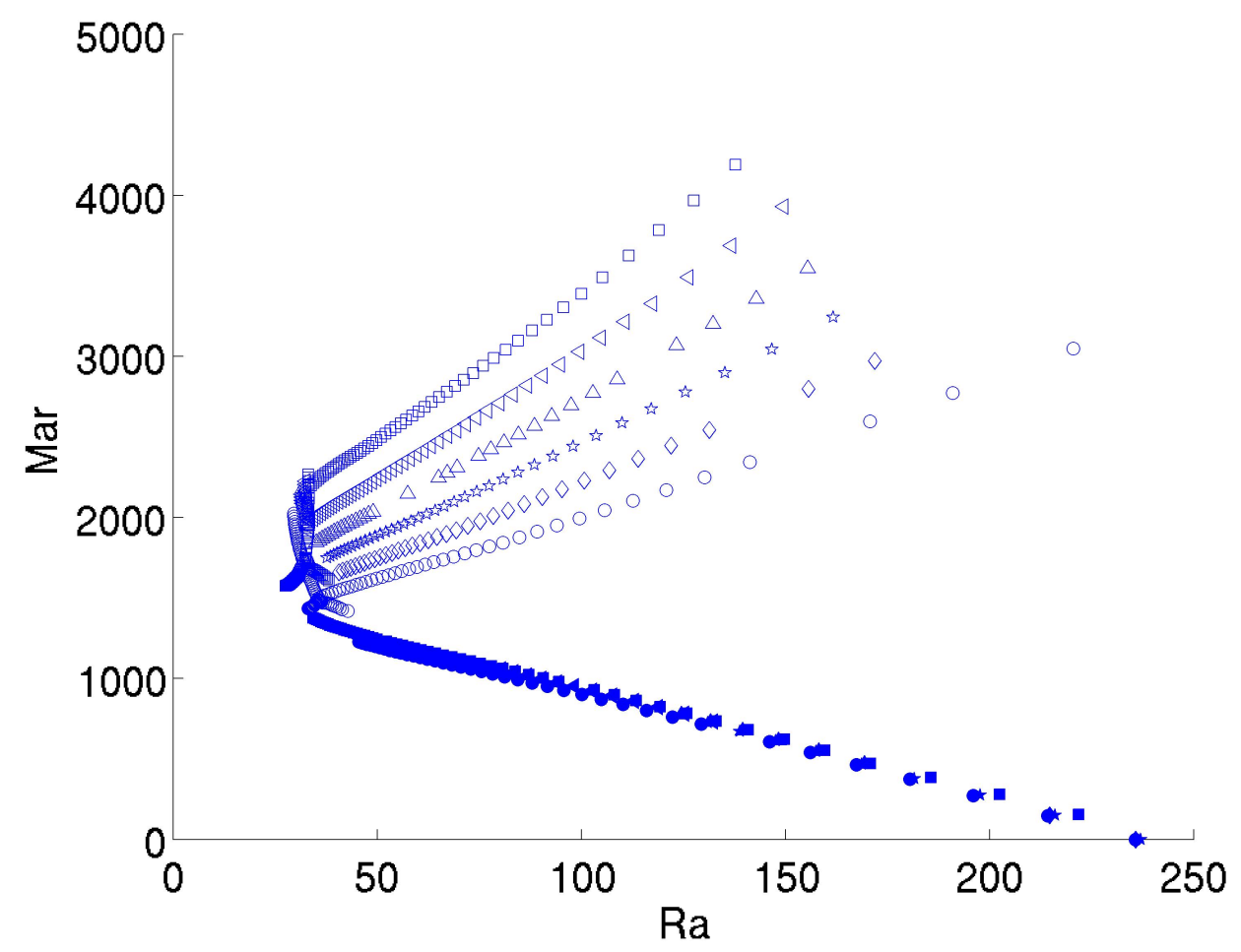

Figure 11: Location in the $\mathrm{Ra}$ - Mar plane of the codimension two-points found for $\operatorname{Pr}=0.7,0.8,0.9,1.0,1.1,1.2$ (circles, diamonds, stars, down triangles, left triangles and squares). Bold points corresponds to the curves separating regions LR and HWI in figure 9 for each Prandtl number. These points seem to be independent of Prandtl number

solved by using spectral methods. The main control parameters were the Marangoni and Rayleigh numbers, related by the Bond number, the Prandtl number, and the Biot number.

It has been confirmed that the main parameter controlling the shape of the basic state is the Bond number with little or null effect coming from Biot or Prandtl numbers. A codimension four point has been found for the first time in this geometry, as an intersection of several codimension two curves. For high Prandtl numbers, it has been possible to identify the range of Bond numbers where the main effect is buoyancy or thermocapillarity. Several new features are of interest in the case of low Prandtl numbers. First, for Biot 
greater than one and Bond below thirty, it has been found that the critical pairs $\left(\mathrm{Ra}_{c}, \mathrm{Mar}_{c}\right)$ separating the regions HWI and LR are almost independent of the Prandtl number 11. Secondly, for small Biot number, it was found [12] that for low Biot the growing solution was of the SR class. In this case it has been always possible to find a Bond number such that the perturbation is an hydrothermal wave, changing completely the behavior of the system.

A new question arises. As the Bond number is defined as $\mathrm{Bo}=\mathrm{Ra} / \mathrm{Ma}=$ $g \alpha \rho d^{2} / \gamma$, what happens when the geometry is changed, and thus transforming the aspect ratio of the box. The idea behind this is to find a new geometry such that the transition between thermocapillarity and thermo-buoyancy would be sharper, in order to better identify the structure coming from these effects.

\section{Symbols and constants}




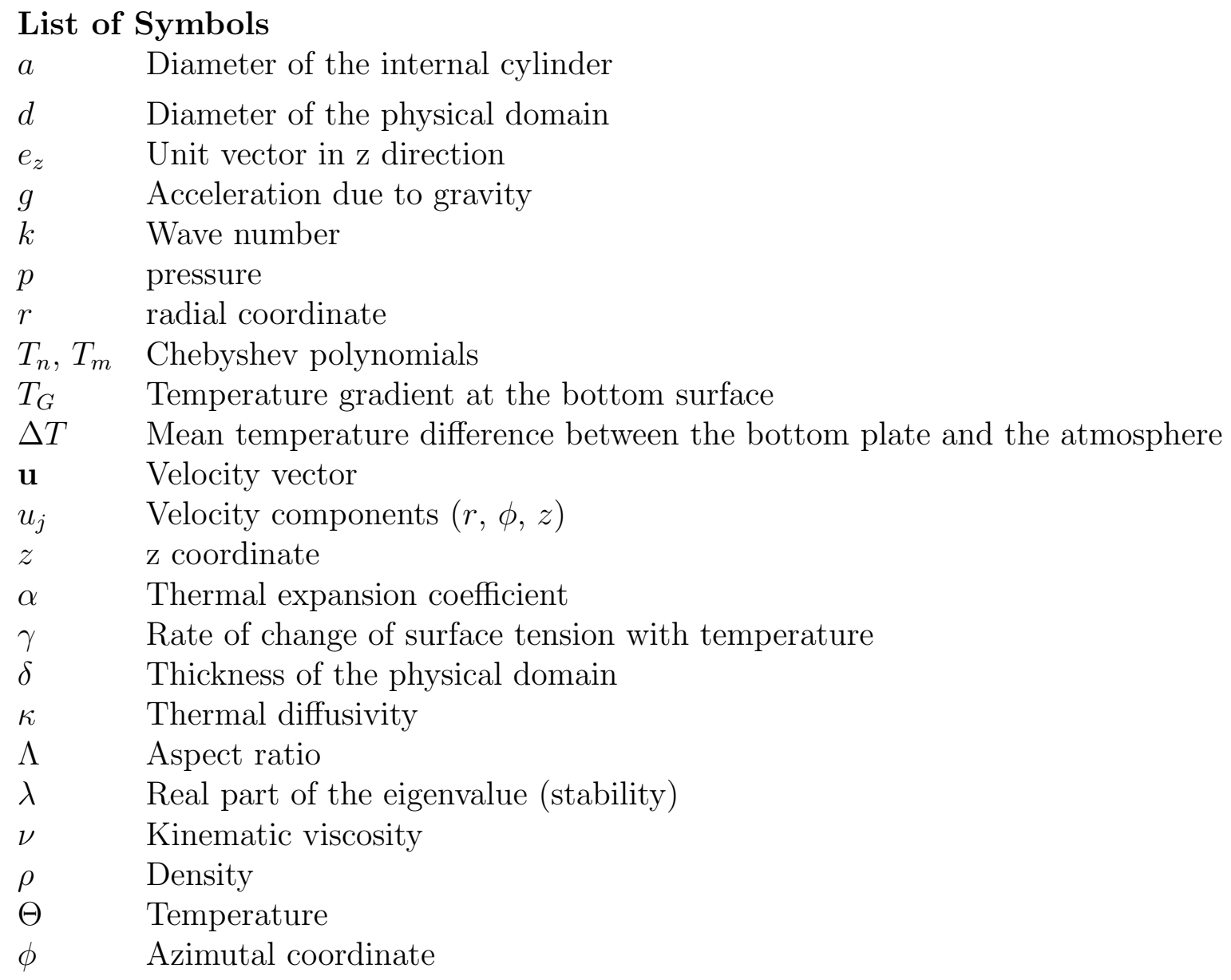

\section{Dimensionless numbers}

Bi Biot Number

Bo Bond number, Bo $=\mathrm{Ra} / \mathrm{Mar}=g \alpha \rho d^{2} / \gamma$

Mar Marangoni number, Mar $=\gamma \Delta T d^{2} / \rho \kappa \nu$

$\operatorname{Pr} \quad$ Prandtl number, $\operatorname{Pr}=\nu / \kappa$

Ra Rayleigh number, $\mathrm{Ra}=g \alpha \Delta T d^{4} / \kappa \nu$

\section{Sub- and Superscripts}

c Critical value 


\section{Acknowledgements}

The authors would like to thank Mr. Darío Hoyas and Ms. Amelia Gil for fruitful conversations about the paper. This work was supported by a generous grant of computer time from the supercomputing center of the UPV.

\section{Bibliography}

[1] H. Bènard. Les tourbillons cellulaires dans une nappe liquide. Rev. Gen. Sci. Pure Appl., 11:1261-1271, 1900.

[2] C. Bernardi and Y. Maday. Approximations spectrales des problmes aux limites elliptiques. Springer-Verlag, 1992.

[3] J. Burguete, N. Mukolobwiez, F. Daviaud, N. Garnier, and A. Chiffaudel. Buoyant-thermocapillary instabilities in extended liquid layers subjected to a horizontal temperature gradient. Physics of Fluids, 13(10):2773-2787, 2001.

[4] C. Canuto, M.Y. Hussaini, A. Quarteroni, and T.A. Zang. Spectral Methods in Fluid Dynamics. Springer-Verlag, 1988.

[5] S. Chandrasekhar. Hydrodynamic and Hydromagnetic Stability. Dover Publications, 1961.

[6] F. Daviaud and J.M. Vince. Traveling waves in a fluid layer subjected to a horizontal temperature gradient. Physical Review E, 48(6):4432-4436, 1993.

[7] E.R.G. Eckert, R.J. Goldstein, W.E. Ibele, S.V. Patankar, T.W. Simon, T.H. Kuehn, P.J. Strykowski, K.K. Tamma, A. Bar-Cohen, J.V.R. Heberlein, J.H. Davidson, J. Bischof, F.A. Kulacki, U. Kortshagen, and S. Garrick. Heat transfer - a review of 1997 literature. International Journal of Heat and Mass Transfer, 43(14):2431-2528, 2000.

[8] A.B. Ezersky, A. Garcimartín, J. Burguete, H.L. Mancini, and C. PrezGarca. Hydrothermal waves in marangoni convection in a cylindrical container. Physical Review E, 47(2):1126-1131, 1993. 
[9] E. Favre, L. Blumenfeld, and F. Daviaud. Instabilities of a liquid layer locally heated on its free surface. Physics of Fluids, 9(5):1473-1475, 1997.

[10] N. Garnier and A. Chiffaudel. Two dimensional hydrothermal waves in an extended cylindrical vessel. European Physical Journal B, 19(1):8795, 2001.

[11] H. Herrero and A.M. Mancho. Influence of aspect ratio in convection due to nonuniform heating. Physical Review E - Statistical Physics, Plasmas, Fluids, and Related Interdisciplinary Topics, 57(6):7336-7339, 1998.

[12] S. Hoyas, A. Gil, P. Fajardo, and M.J. Pérez-Quiles. Co-dimension Three Bifurcations in a Bénard-Marangoni Problem. Physical Review E, 88(015001), 2013.

[13] S. Hoyas, H. Herrero, and A. M. Mancho. Thermocapillar and thermogravitatory waves in a convection problem. Theoretical and Computational Fluid Dynamics, 18(2-4):309-321, 2004.

[14] S. Hoyas, H. Herrero, and A.M. Mancho. Bifurcation diversity of dynamic thermocapillary liquid layers. Physical Review E - Statistical, Nonlinear, and Soft Matter Physics, 66(5):057301/1-057301/4, 2002.

[15] S. Hoyas, H. Herrero, and A.M. Mancho. Thermal convection in a cylindrical annulus heated laterally. Journal of Physics A: Mathematical and General, 35(18):4067-4083, 2002.

[16] S. Hoyas, H. Herrero, A.M. Mancho, N. Garnier, and A. Chiffaudel. Bénard-Marangoni convection in a differentially heated cylindrical cavity. Phys. Fluids, 1(7):054104, 2005.

[17] J. Jiménez and S. Hoyas. Turbulent fluctuations above the buffer layer of wall-bounded flows. Journal of Fluid Mechanics, 611:215-236, 2008.

[18] A.M. Mancho and H. Herrero. Instabilities in a laterally heated liquid layer. Physics of Fluids, 12(5):1044-1051, 2000. 
[19] A.M. Mancho, H. Herrero, and J. Burguete. Primary instabilities in convective cells due to nonuniform heating. Physical Review E - Statistical Physics, Plasmas, Fluids, and Related Interdisciplinary Topics, 56(3 SUPPL. A):2916-2923, 1997.

[20] J.F. Mercier and C. Normand. Buoyant-thermocapillary instabilities of differentially heated liquid layers. Physics of Fluids, 8(6):1433-1445, 1996.

[21] M.C. Navarro, H. Herrero, and S. Hoyas. Chebyshev collocation for optimal control in a thermoconvective flow. Communications in Computational Physics, 5(2-4):649-666, 2009.

[22] Steven A. Orszag. Comparison of pseudospectral and spectral approximation. Studies in Applied Mathematics, 51(3):253-259, 1972.

[23] S.M. O'Shaughnessy and A.J. Robinson. Heat transfer near an isolated hemispherical gas bubble: The combined influence of thermocapillarity and buoyancy. International Journal of Heat and Mass Transfer, 62(1):422-434, 2013.

[24] M.A. Pelacho and J. Burguete. Temperature oscillations of hydrothermal waves in thermocapillary-buoyancy convection. Physical Review E Statistical Physics, Plasmas, Fluids, and Related Interdisciplinary Topics, 59(1):835-840, 1999.

[25] L. Peng, Y.-R. Li, W.-Y. Shi, and N. Imaishi. Three-dimensional thermocapillary-buoyancy flow of silicone oil in a differentially heated annular pool. International Journal of Heat and Mass Transfer, 50(56):872-880, 2007.

[26] R.J. Riley and G.P. Neitzel. Instability of thermocapillary-buoyancy convection in shallow layers. Part 1. Characterization of steady and oscillatory instabilities. Journal of Fluid Mechanics, 359:143-164, 1998.

[27] W.Y. Shi, M.K. Ermakov, Y.-R. Li, L. Peng, and N. Imaishi. Influence of buoyancy force on thermocapillary convection instability in the differentially heated annular pools of silicon melt. Microgravity Science and Technology, 21(SUPPL. 1):S289-S297, 2009. 
[28] W.Y. Shi, X. Liu, G. Li, Y.-R. Li, L. Peng, M.K. Ermakov, and N. Imaishi. Thermocapillary convection instability in shallow annular pools by linear stability analysis. Journal of Superconductivity and Novel Magnetism, 23(6):1185-1188, 2010.

[29] Marc K. Smith and Stephen H. Davis. Instabilities of dynamic thermocapillary liquid layer. part 1. convective instabilities. Journal of Fluid Mechanics, 132:119-144, 1983.

[30] A.J. Torregrosa, S. Hoyas, M.J Perez-Quiles, and J.M. Mompo-Laborda. Bifurcation diversity in an annular pool heated from below: Prandtl and biot numbers effects. Communications in Computational Physics, 13(2):428-441, 2013. 